\title{
Associations between added sugars and micronutrient intakes and status: further analysis of data from the National Diet and Nutrition Survey of Young People aged 4 to 18 years
}

\author{
Sigrid Gibson ${ }^{1 *}$ and Alison Boyd ${ }^{2}$ \\ ${ }^{1}$ Sig-Nurture Ltd, 11 Woodway, Guildford, Surrey GU1 2TF, UK \\ ${ }^{2}$ Sugar Bureau, 6 Catherine Street, London WC2B 5JJ, UK
}

(Received 19 September 2007 - Revised 19 March 2008 - Accepted 20 March 2008 - First published online 8 July 2008)

Added sugars are often viewed as 'empty calories', negatively impacting micronutrient intakes, yet reviews consider the evidence inconclusive. This study aimed to quantify associations between dietary added sugars (as a percentage of energy) and micronutrient intake and biochemical status in the National Diet and Nutrition Survey. Using data from 1688 British children aged 4-18 years who completed $7 \mathrm{~d}$ weighed dietary records in 1997, micronutrient intakes were examined across quintiles of added sugars. After excluding low energy reporters, mean dietary intakes of most nutrients exceeded the reference nutrient intake, except for zinc. Compared with quintile 1 ( $9 \%$ added sugars), high consumers in quintile 5 (23\% added sugars) had micronutrient intakes ranging from $24 \%$ lower to $6 \%$ higher (mean $14 \%$ lower). Zinc intakes in quintile $1 v$. quintile 5 averaged $93 \%$ v. $78 \%$ of reference nutrient intake; magnesium $114 \%$ v. $94 \%$; iron $115 \%$ v. $100 \%$; and vitamin A $111 \%$ v. $92 \%$, respectively. Plasma levels of magnesium, zinc and carotenoids did not vary across quintiles, but weak negative correlations were observed with serum ferritin and transferrin saturation. Plasma selenium was inversely correlated with added sugars $(r-0 \cdot 17 ; P<0 \cdot 0001)$ but there was no association with glutathione peroxidase. The impact of added sugars on micronutrient intakes appears modest overall but may have relevance for children consuming inadequate amounts of nutrient-rich foods coupled with a diet high in added sugars (approximately $23 \%$ ). Further work is needed to explore the impact of different sources of added sugars and to refine assessments of inadequate intakes and status.

Added sugars: Micronutrient intake: Biochemical status: Children

Over thirty observational studies and four small interventions have explored the concept that added sugars dilute the micronutrient content of the diet. Several reviews have concluded that evidence for micronutrient dilution is generally inconsistent, and that where associations are found, these are weak ${ }^{(1-7)}$. Furthermore, relationships between sugars and micronutrients may not be nutritionally meaningful in a context where intakes of most nutrients are adequate. However, these reviews also acknowledge that some inconsistencies may be due to methodological differences. These include different definitions of sugars (e.g. total sugars, non-milk extrinsic sugars (NMES) or added sugars), adjustment for energy intake (EI) and confounding factors such as misreporting error. It is therefore important that research should attend to confounding factors and attempt to quantify the observed associations to provide a better evidence base for dietary recommendations ${ }^{(6,7)}$.

Associations have previously been reported between micronutrient intakes and the proportion of sugars in the diet among young children, adults and older people in Britain ${ }^{(8-12)}$. Some studies included an examination of status indices to help validate the dietary assessment and evaluate the biochemical significance of any dilution effect. This new analysis of data from the National Diet and Nutrition Survey of Young People aged 4 to 18 years $(\mathrm{NDNS} 4-18)^{(13)}$ examines the strength of association between micronutrients and the proportion of added sugars in the diets and the likely significance of this for nutritional health in this age group. It includes an evaluation of the impact of low reported EI. Added sugars were chosen as the exposure variable rather than NMES because the latter definition includes fruit juices, which may give rise to positive associations with some nutrients such as vitamin $\mathrm{C}$.

\section{Methods \\ Survey method}

The NDNS4-18 consists of a nationally representative sample of 2672 young people aged 4-18 years, randomly sampled from 132 postcode sectors throughout mainland Britain in 1997. The survey fieldwork spanned a full calendar year. Only one young person per household was recruited. An interview was conducted to provide information on socio-demographic circumstances, medication use, and eating and drinking habits. Each young person or carer was supplied

Abbreviations: EAR, estimated average requirement; \%EAS, percentage of energy from added sugars; EI, energy intake; LER, low energy reporters; NDNS4-18, National Diet and Nutrition Survey of Young People aged 4 to 18 years; NMES, non-milk extrinsic sugars; Q1-Q5, quintiles 1-5; RNI, reference nutrient intake. * Corresponding author: Mrs Sigrid Gibson, fax +44 1483 838018, email sigrid@sig-nurture.com 
with a set of digital food scales and two recording diaries; the 'home record' diary for foods eaten in the home and the 'eating out' diary for foods that were eaten outside the home and could not be weighed. A description was recorded of each food or drink item consumed over the $7 \mathrm{~d}$, including brand name and method of preparation. Ethical approval for the survey was obtained from National Health Services Local Research Ethics Committees.

\section{Data analysis}

Data files were obtained from the UK Data Archive (www.data-archive.ac.uk). Of the 1701 completed $7 \mathrm{~d}$ dietary records, thirteen records were rejected due to missing data (zeros) for nutrient sources, leaving a sample of 1688. Values for NMES intake were used to estimate added sugars intake (from total NMES intake minus NMES from fruit juices). The percentage of energy from added sugars (\%EAS) for each individual was calculated as: Added sugars $(\mathrm{g}) \times 3.75 \times 100 / \mathrm{EI}(\mathrm{kcal})$.

Quintile cut-offs were generated for each age group (4-6, $7-10,11-14,15-18$ years) in boys and girls separately. These groups were then amalgamated to provide quintiles matched for age and sex. Mean intakes of EAS across the five quintiles (Q1-Q5) were 9, 12, 15, 18 and $23 \%$.

From survey data on weight and height, BMI $z$-scores were calculated based on UK reference curves ${ }^{(14)}$. Children were classified as 'overweight/obese' using International Obesity Task Force cut-offs for BMI $z$-scores of 1.3 (boys) and 1.19 (girls); these correspond to the adult criteria of BMI $>25 \mathrm{~kg} / \mathrm{m}^{2}$, extrapolated back to childhood ${ }^{(15-17)}$.

\section{Adjustment for low energy reporting}

Previous work has shown that children in this survey who recorded relatively low EI compared to their predicted BMR reported eating diets lower in sugars $(\% \text { energy })^{(18)}$. Since this may attenuate any inverse association between sugars and micronutrient intake, the impact of excluding low energy reporters (LER) was explored using a cut-off for EI:BMR of $1 \cdot 2$, below which records are not considered a plausible measure of food consumed during the survey period $^{(19)}$. While cut-offs lack good sensitivity and specificity for identifying individual under-reporters ${ }^{(20)}$, they are adequate for exploring how an under-reporting bias might influence trends.

\section{Statistical methods}

Differences between quintile groups in regard to under-reporting, social class, ethnicity, dieting and illness during the survey were examined by cross-tabulation and $\chi^{2}$ tests. Trends in micronutrient intakes across quintiles were examined visually using error bar charts (mean and $95 \% \mathrm{CI}$ ), both before and after excluding LER. Differences between groups were evaluated using ANOVA and contrast tests (with correction for unequal variances and Bonferroni adjustment for multiple comparisons). Individuals' intakes were expressed as a percentage of their reference nutrient intake (RNI) and estimated average requirement $(\mathrm{EAR})^{(21)}$ to identify which nutrients were most marginal in the diet. The prevalence of inadequate intakes within a group was estimated as the percentage of the population with usual intakes below the $\mathrm{EAR}^{(22)}$.

\section{Micronutrient status}

A total of 1075 children provided a fasting blood sample (59\% of the sample completing $7 \mathrm{~d}$ diaries). Distributions of status measures were examined for normality and the presence of extreme values. One outlier with serum ferritin of $990 \mathrm{~g} / \mathrm{l}$ (indicative of infection/inflammation) was excluded, since other serum analytes can be distorted by an acute phase response. All other values were under the normal upper limit of $300 \mathrm{~g} / \mathrm{l}$. Several analytes (serum ferritin and the plasma carotenoids) were positively skewed (as indicated by skewness estimates greater than +1 ). For these, log-transformed values $(\log n)$ were used in analysis. One-way ANOVA and post hoc tests for contrast (Q1 v. Q5) were used to assess differences in status by quintile of \%EAS. Partial correlations between variables (adjusted for sex and age) were examined.

Three indices commonly used in population screening were selected for assessment of iron status. The prevalence of low iron status was compared across quintiles of \%EAS using $\chi^{2}$ tests. Prevalence criteria for low iron status were based on the definitions used in the NDNS report ${ }^{(13)}$, as follows. Anaemia: $\mathrm{Hb}<110 \mathrm{~g} / \mathrm{l}$ for children under 6 years, $\mathrm{Hb}<120 \mathrm{~g} / \mathrm{l}$ for girls age $6-18$ and boys age $6-15$ years, $\mathrm{Hb}<130 \mathrm{~g} / \mathrm{l}$ for boys over 15 years; low iron stores: serum ferritin $<15 \mu \mathrm{g} / \mathrm{l}$ for girls, $<20 \mu \mathrm{g} / \mathrm{l}$ for boys; low transferrin saturation: $<15 \%$.

All methods of blood analysis and quality control are described in Appendix $Q$ of the published survey report ${ }^{(13)}$.

\section{Results}

The mean intake of added sugars as \%EAS was 15.4 (SD 5.3) \% (Fig. 1) with Q1-Q5 means of 9, 12, 15, 18 and $23 \%$ (Table 1). The corresponding intakes of NMES across the quintiles were about one percentage point higher than those of added sugars. Thus, Q1 corresponds closely to the recommended population level of $10 \%$ total energy from NMES ${ }^{(21)}$, while Q5 is close to the maximum level of added sugars suggested by the American Institute of Medicine report ${ }^{(23)}$. Soft drinks (including fruit squash but not fruit juice) contributed $4.4 \%$ of energy overall or about $28 \%$ of added sugars.

Young people with the lowest sugar intake (Q1) were more likely to be overweight (BMI $>25 \mathrm{~kg} / \mathrm{m}^{2}$ ), or dieting, or to report an EI below the plausible threshold (EI:BMR $<1 \cdot 2$; Table 1). There were slightly more non-Caucasians in this group, but no other significant differences in regard to social class, vegetarianism or illness that affected eating during the survey.

\section{Micronutrient adequacy}

In the total sample, intakes of micronutrients tended to be highest in Q2 (mean 12\% EAS) and lowest in Q5 (mean $23 \%$ EAS) for all nutrients except vitamin C (Table 2). Vitamin $\mathrm{C}$ showed no relationship with the level of added sugars in the diet, largely due to fruit drinks (squash etc.) being a major source of both sugar and vitamin $\mathrm{C}$ for these 


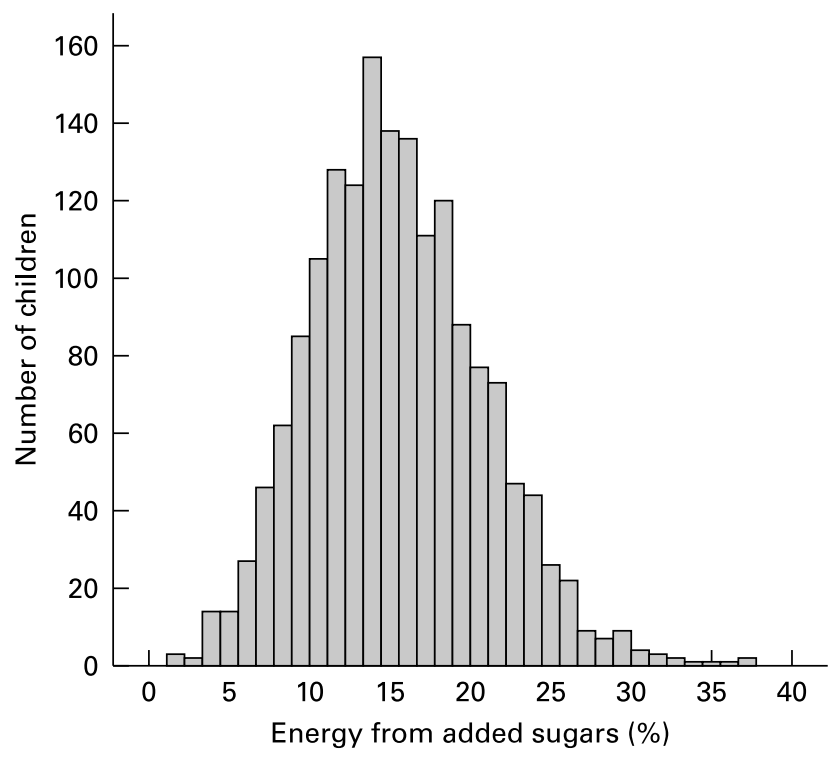

Fig. 1. Frequency distribution of added sugars (as a percentage of total energy intake) ( $n$ 1688).

children. Mean intakes of iron, magnesium, zinc and vitamin A in the total sample were $97,92,82$ and $97 \%$ of the RNI, respectively (data not shown). Calcium, folate and riboflavin were between 110 and $150 \%$ of the RNI, while intakes of other nutrients (thiamin, niacin, vitamin $\mathrm{B}_{6}$, vitamin $\mathrm{B}_{12}$, vitamin $\mathrm{C}$, vitamin $\mathrm{E}$ ) were well above recommended levels at all levels of added sugars.

\section{Impact of low energy reporting}

Exclusion of LER (i.e. confining the analysis to children with plausible records or EI:BMR $>1.2$ ) gave associations that were more linear but at higher levels of adequacy.

Zinc intakes were below the RNI at all levels of added sugars (Table 3). There was a downward trend in intakes of most nutrients with increasing added sugars intake such that intakes in Q5 were 10-20\% lower than in Q1 (14-18\% lower for the four 'marginal' minerals: iron, calcium, magnesium and zinc). However, vitamin $\mathrm{B}_{6}$ and vitamin $\mathrm{C}$ showed no trend with sugars intake and vitamin $\mathrm{E}$ showed a weak positive association. At the highest level of added sugars (Q5), mean intakes of zinc, magnesium and vitamin A fell below the RNI (Fig. 2). The likely prevalence of inadequacy, as estimated by the percentage of children with intakes below the EAR, was significantly higher in Q5 compared with Q1 for the five nutrients most at risk (iron, calcium, magnesium, zinc and vitamin A) (Table 4). Few children had intakes below the lower RNI, although in Q5, $11 \%$ had magnesium intakes and $16 \%$ had zinc intakes below this threshold. Energy intake, which is recognised as the main determinant of nutrient adequacy ${ }^{(21,24)}$ rose by approximately $6 \%$ from Q1 to Q5.

\section{Food intake}

Unsurprisingly, given the classification of respondents according to the proportion of added sugars in their diet, consumption of most other food groups declined across Q1-Q5 (Table 5). In particular, consumption of fruit juice, fruit, vegetables, meat, bread, fats, cheese and eggs fell by more than $20 \%$. Around $3-5 \%$ of the variance in intake of these food groups was explained by the level of added sugars in the diet.

\section{Micronutrient status}

Associations between sugars intake and selected micronutrient status indices for the total sample are shown in Table 6. Exclusion of LER weakened the power to detect significant associations but did not alter the direction of associations. Serum ferritin was $12-17 \%$ higher among low-sugar consumers (Q1) compared with consumers in other quintiles $(P=0.03)$ while transferrin values showed a smaller difference $(24 \%$ in Q1 v. $22 \%$ in Q5, $P=0.046)$. However, there was no significant rise in the prevalence of low iron status with a higher sugars intake (data not shown). Plasma selenium declined by around $8 \%$ (mean $0.9 \mu \mathrm{mol} / \mathrm{l}$ in Q1 v. $0.83 \mu \mathrm{mol} / 1$ in $\mathrm{Q} 5 ; r-0.17, P<0.0001)$. Overall $13 \%$ of

Table 1. Background differences for young people according to added sugars intake ( $n$ 1688)*

\begin{tabular}{|c|c|c|c|c|c|c|}
\hline & \multicolumn{5}{|c|}{ Quintiles of $\%$ added sugars (\%EAS) $\dagger$} & \multirow[b]{2}{*}{$\chi^{2} P$ value } \\
\hline & Q1 (9\%) & Q2 (12\%) & Q3 (15\%) & Q4 (18\%) & Q5 (23\%) & \\
\hline \multicolumn{7}{|l|}{ Ethnic group (\%) } \\
\hline White & 86 & 91 & 92 & 92 & 95 & 0.002 \\
\hline Other & 14 & 9 & 8 & 8 & 5 & \\
\hline \multicolumn{7}{|l|}{ Social class (\%) } \\
\hline Non-manual & 45 & 49 & 45 & 50 & 49 & 0.50 \\
\hline Manual & 55 & 51 & 55 & 50 & 51 & \\
\hline Dieting to lose weight (\%) & 8 & 4 & 4 & 4 & 2 & 0.003 \\
\hline Vegetarian or vegan (\%) & 3 & 4 & 3 & 2 & 3 & 0.36 \\
\hline Illness affecting eating (\%) & 10 & 11 & 10 & 10 & 13 & 0.70 \\
\hline Low energy reporters ( $\%$ with El:BMR $<1 \cdot 2)$ & 42 & 28 & 26 & 21 & 19 & 0.0001 \\
\hline Overweight or obese (\%)‡ & 29 & 22 & 20 & 17 & 17 & 0.0001 \\
\hline
\end{tabular}

* For details of procedures, see Methods.

$\dagger$ Age/sex-adjusted quintiles of percentage energy from added sugars (\%EAS).

† BMI $z$-score: $>1.3$ (boys), $>1.19$ (girls). 
Table 2. Energy and micronutrient intakes according to added sugars intake $(n 1688)^{\star}$

\begin{tabular}{|c|c|c|c|c|c|c|}
\hline & \multicolumn{5}{|c|}{ Quintiles of $\%$ added sugars } & \multirow[b]{2}{*}{ Mean } \\
\hline & Q1 & Q2 & Q3 & Q4 & Q5 & \\
\hline$n$ & 336 & 338 & 339 & 339 & 336 & \\
\hline Energy (kJ) & 6838 & 7237 & 7440 & 7521 & 7527 & 7313 \\
\hline Added sugars ( $\mathrm{g} / \mathrm{d}$, calculated) & 38 & 57 & 71 & 86 & 111 & 72 \\
\hline Energy from added sugars (\%) & 9 & 12 & 15 & 18 & 23 & 15 \\
\hline Energy from NMES (\%) & 10 & 14 & 16 & 19 & 24 & 17 \\
\hline Calcium (mg) & 701 & 745 & 751 & 726 & 691 & 723 \\
\hline Magnesium (mg) & 198 & 201 & 198 & 192 & 184 & 195 \\
\hline Phosphorus (mg) & 1015 & 1043 & 1035 & 1013 & 963 & 1014 \\
\hline Iron (mg) & 9.5 & 9.7 & $9 \cdot 6$ & $9 \cdot 5$ & $9 \cdot 0$ & 9 \\
\hline Copper (mg) & 0.82 & 0.83 & 0.83 & 0.84 & 0.78 & 1 \\
\hline Zinc (mg) & $6 \cdot 6$ & $6 \cdot 5$ & $6 \cdot 4$ & $6 \cdot 4$ & $5 \cdot 7$ & 6 \\
\hline Retinol $(\mu \mathrm{g})$ & 334 & 332 & 344 & 311 & 261 & 316 \\
\hline Carotene $(\mu \mathrm{g})$ & 1413 & 1475 & 1384 & 1324 & 1294 & 1378 \\
\hline Retinol equivalents & 537 & 538 & 542 & 502 & 446 & 513 \\
\hline Vitamin $D(\mu \mathrm{g})$ & 2.7 & $2 \cdot 7$ & $2 \cdot 6$ & $2 \cdot 4$ & $2 \cdot 2$ & 2.5 \\
\hline Thiamin (mg) & 1.5 & 1.5 & 1.6 & 1.5 & $1 \cdot 3$ & 1.5 \\
\hline Riboflavin (mg) & 1.5 & 1.6 & 1.6 & 1.6 & 1.5 & $1 \cdot 6$ \\
\hline Niacin equivalents (mg) & 28 & 28 & 27 & 27 & 24 & $26 \cdot 6$ \\
\hline Vitamin C (mg) & 75 & 81 & 77 & 79 & 81 & 78.6 \\
\hline Vitamin E (mg) & $8 \cdot 4$ & $8 \cdot 2$ & $8 \cdot 8$ & $8 \cdot 4$ & $7 \cdot 6$ & $8 \cdot 3$ \\
\hline Vitamin $B_{6}(\mathrm{mg})$ & $2 \cdot 0$ & $2 \cdot 0$ & $2 \cdot 0$ & $2 \cdot 1$ & $1 \cdot 8$ & $2 \cdot 0$ \\
\hline Vitamin $B_{12}(\mu \mathrm{g})$ & $4 \cdot 0$ & $4 \cdot 0$ & $4 \cdot 0$ & $4 \cdot 0$ & $3 \cdot 5$ & $3 \cdot 9$ \\
\hline Folate $(\mu \mathrm{g})$ & 230 & 234 & 223 & 213 & 195 & 219 \\
\hline NSP fibre (g) & $11 \cdot 0$ & $11 \cdot 2$ & $10 \cdot 6$ & 9.9 & $9 \cdot 3$ & $10 \cdot 4$ \\
\hline
\end{tabular}

NMES, non-milk extrinsic sugars.

${ }^{*}$ For details of procedures and quintiles, see Methods and Table 1.

children (18\% in Q5) had levels below the reference range for age $4-16$ years $(0.7-1.7 \mu \mathrm{mol} / \mathrm{l})^{(25)}$ although none had levels indicative of frank selenium deficiency. There was no evidence of any association between \%EAS and glutathione peroxidase (data not shown). Plasma zinc did not vary with added sugar intake and levels were consistently above the recognised deficiency level. There was a weak positive association with $\alpha$-cryptoxanthin $(P=0.003)$ but no association with other carotenoids.
Correlations of these status measures with food intakes were also examined (data not shown). Serum ferritin was positively correlated with meat consumption $(r+0 \cdot 21, P<0 \cdot 0001)$ with weaker associations for $\mathrm{Hb}(r+0 \cdot 18, P<0.0001)$ and transferrin saturation $(r+0 \cdot 11, P<0 \cdot 001)$. Selenium status was positively associated with intake of fish $(r+0.23, P<0.0001)$, cereal dishes (pasta/rice/pizza) $(r+0.23, P<0.0001)$, eggs $(r+0.15, \quad P<0.0001)$ and meat $(r+0.16, \quad P<0.0001)$, but inversely with savoury snacks $(r-0.15, P<0.0001)$.

Table 3. Mean micronutrient intakes as percentage of reference nutrient intake (RNI), according to added sugars intake ( $n$ 1217, sample excludes low energy reporters)*

\begin{tabular}{|c|c|c|c|c|c|c|c|c|c|}
\hline & \multicolumn{5}{|c|}{ Quintiles of $\%$ added sugars } & \multirow[b]{2}{*}{ Mean } & \multirow[b]{2}{*}{ \% Difference Q5 v. Q1 } & \multicolumn{2}{|c|}{ Contrast $P$ value } \\
\hline & Q1 & Q2 & Q3 & Q4 & Q5 & & & Q5 v. 1 & Q3 v. 1 \\
\hline$n$ & 190 & 240 & 249 & 268 & 270 & & & & \\
\hline \multicolumn{10}{|c|}{ Percentage of RNI } \\
\hline Calcium & 133 & 132 & 127 & 121 & 114 & 125 & -14 & $<0.001$ & 0.253 \\
\hline Magnesium & 114 & 108 & 103 & 99 & 94 & 103 & -18 & $<0.001$ & 0.002 \\
\hline Iron & 115 & 110 & 107 & 106 & 100 & 107 & -14 & $<0.001$ & 0.022 \\
\hline Zinc & 93 & 91 & 88 & 86 & 78 & 87 & -16 & $<0.001$ & 0.025 \\
\hline Vitamin A & 111 & 109 & 107 & 102 & 92 & 104 & -18 & $<0.001$ & 0.421 \\
\hline Thiamin & 221 & 209 & 219 & 194 & 182 & 204 & -17 & $<0.001$ & 0.866 \\
\hline Riboflavin & 171 & 175 & 165 & 163 & 154 & 165 & -10 & 0.004 & 0.306 \\
\hline Niacin & 246 & 235 & 221 & 222 & 204 & 224 & -17 & $<0.001$ & $<0.001$ \\
\hline Vitamin $B_{6}$ & 225 & 231 & 231 & 233 & 234 & 231 & 4 & 0.105 & 0.269 \\
\hline Vitamin $\mathrm{B}_{12}$ & 427 & 417 & 410 & 401 & 353 & 400 & -17 & $<0.001$ & 0.326 \\
\hline Folate & 170 & 159 & 150 & 143 & 130 & 149 & -24 & $<0.001$ & 0.001 \\
\hline Vitamin C & 243 & 240 & 229 & 246 & 241 & 240 & -1 & 0.915 & 0.338 \\
\hline Vitamin E† & 176 & 177 & 178 & 179 & 186 & 179 & 6 & 0.002 & 0.429 \\
\hline
\end{tabular}

${ }^{*}$ For details of procedures and quintiles, see Methods and Table 1.

† Calculated as $0.4 \mathrm{mg} / \mathrm{g}$ dietary PUFA. 


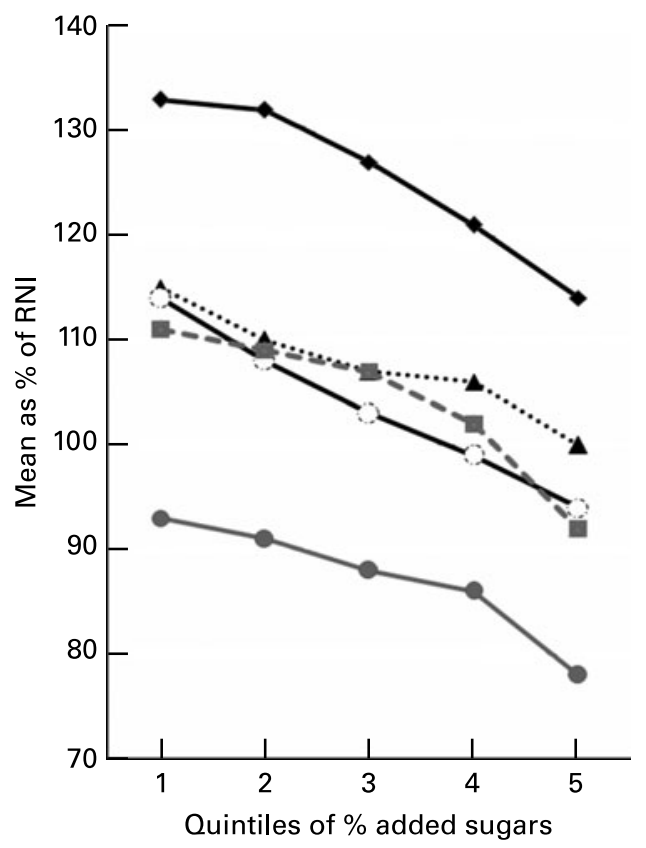

Fig. 2. Trends in intake of selected nutrients according to added sugars ( $n 1217$ excluding low energy reporters). $\bullet$, Calcium; $\boldsymbol{\Lambda}$, iron; $O$, magnesium; , vitamin $\mathrm{A} ; \boldsymbol{\ominus}$, zinc. RNI, reference nutrient intake.

With the exception of selenium status, the correlation coefficients were larger with these foods than with \%EAS.

\section{Discussion}

A major strength of the present study is the dietary methodology ( $7 \mathrm{~d}$ weighed record), which is better able to estimate the prevalence of inadequacy than $24 \mathrm{~h}$ recall methods used in many large-scale epidemiological studies. Estimated BMR values also allowed us to examine the impact of LER bias, which has not always been possible in other studies. Limitations of the NDNS include a moderate sample size ( $n$ 1688, or $n 1217$ after exclusion of LER), which limited the ability to stratify the sample further. Blood samples were only available for around 1000 children, also limiting the statistical power to detect associations with nutrient status. Selenium intake data were also absent due to insufficient analytical data.

Prior to adjustment for LER, associations with some nutrients (calcium, iron, magnesium, zinc, riboflavin and folate) were curvilinear, with highest values in Q2 (12\% added sugars) and Q3 (15\% added sugars). A similar pattern has been found in the literature ${ }^{(1,2,9,26)}$, as summarised by Ruxton et al. ${ }^{(3)}$. However, on exclusion of LER the trends became more linear and levels of nutrient intake were elevated. Rutishauser et al. ${ }^{(27)}$ have pointed out that failure to correct for implausible energy values may lead to misleading (overinflated) estimates of inadequacy in populations.

We have attempted to quantify the micronutrient dilution observed in the present study. Overall, a 2.5-fold difference in sugars concentration (23\% in Q5 v. $9 \%$ in Q1) was associated with a reduction in micronutrient intake (as a percentage of the RNI) of the order of 10-20\% (range -24 to $+6 \%$ ). With respect to iron and calcium, for example, the impact is equivalent to a $1 \%$ reduction relative to the RNI for every percentage point increase in EAS. A recent review ${ }^{(7)}$ found associations which varied between nutrients and between population groups, as well as with the definition of sugars intake. It reasoned that inconsistency in the literature may be due to confounding by EI. For example, in the large CSFII (US) dataset reviewed by the Institute of Medicine ${ }^{(23)}$, there was evidence of a subtle decline in EI at both extremes of the spectrum $(<5 \%$ EAS and $>30 \%$ EAS $)$, which may explain, in part, the observed curvilinear associations with micronutrient intakes ${ }^{(28)}$. In the present study, exclusion of suspiciously low energy records produced estimates of the impact of added sugars on micronutrient and food intakes that should be relatively robust. These indicate that dilution effects start to become significant above Q3 (i.e. $>15 \%$ EAS). Our conclusions are similar to other recent European studies of children in Germany ${ }^{(29)}$ and Norway ${ }^{(30)}$ who consumed a similar range of added sugars.

It is likely that the impact of added sugars on micronutrient intakes depends on the types of sugar-containing foods consumed and whether these contribute nutrients or facilitate consumption of other nutrient-rich foods. For example, consumption of soft drinks, sugar/confectionery, and biscuits

Table 4. Percentage of young people with intakes below the estimated average requirement (EAR) and lower reference nutrient intake (LRNI) by quintile of added sugars ( $n$ 1217, sample excludes low energy reporters)*

\begin{tabular}{|c|c|c|c|c|c|c|c|}
\hline & \multicolumn{5}{|c|}{ Quintiles of $\%$ added sugars } & \multirow[b]{2}{*}{ Mean } & \multirow[b]{2}{*}{ Significant at $P<0.05$} \\
\hline & Q1 & Q2 & Q3 & Q4 & Q5 & & \\
\hline$n$ & 190 & 240 & 249 & 268 & 270 & & \\
\hline Calcium below EAR (\%) & 12 & 8 & 11 & 12 & 17 & 12 & $\mathrm{Q} 5>\mathrm{Q} 2$ \\
\hline Iron below EAR (\%) & 11 & 18 & 22 & 20 & 28 & 20 & $\mathrm{Q} 5, \mathrm{Q} 3,>\mathrm{Q} 1$ \\
\hline Magnesium below EAR (\%) & 16 & 24 & 24 & 30 & 36 & 27 & $\mathrm{Q} 5, \mathrm{Q} 4>\mathrm{Q} 3, \mathrm{Q} 2, \mathrm{Q} 1$ \\
\hline Zinc below EAR (\%) & 23 & 28 & 36 & 36 & 53 & 36 & $\mathrm{Q} 5>\mathrm{Q} 3>\mathrm{Q} 1$ \\
\hline Vitamin A below EAR (\%) & 18 & 22 & 24 & 29 & 31 & 26 & $\mathrm{Q} 5>\mathrm{Q} 1$ \\
\hline Calcium below LRNI (\%) & 0 & 0 & 0 & 0 & 0 & 0 & \\
\hline Iron below LRNI (\%) & 2 & 3 & 4 & 4 & 7 & 4 & $\mathrm{Q} 5>\mathrm{Q} 1$ \\
\hline Magnesium below LRNI (\%) & 5 & 5 & 7 & 8 & 11 & 8 & \\
\hline Zinc below LRNI (\%) & 4 & 5 & 8 & 8 & 16 & 8 & $\mathrm{Q} 5>\mathrm{Q} 3, \mathrm{Q} 2, \mathrm{Q} 1$ \\
\hline Vitamin A below LRNI (\%) & 6 & 4 & 4 & 5 & 8 & 5 & \\
\hline
\end{tabular}

${ }^{*}$ For details of procedures and quintiles, see Methods and Table 1. 
Table 5. Food consumption according to level of added sugars*

\begin{tabular}{|c|c|c|c|c|c|c|c|}
\hline \multirow[b]{2}{*}{ Food consumption (g/d) } & \multicolumn{5}{|c|}{ Quintiles of $\%$ added sugars } & \multirow[b]{2}{*}{ \% Difference Q5 v. Q1 } & \multirow[b]{2}{*}{ ANOVA $P$ for linear trend } \\
\hline & Q1 & Q2 & Q3 & Q4 & Q5 & & \\
\hline Milk & 226 & 233 & 225 & 210 & 198 & -13 & 0.007 \\
\hline Cheese & 11 & 12 & 11 & 10 & 8 & -27 & 0.001 \\
\hline Eggs & 14 & 12 & 9 & 10 & 8 & -42 & $<0.001$ \\
\hline Fat spreads & 13 & 10 & 11 & 9 & 9 & -35 & $<0.001$ \\
\hline Bread & 94 & 79 & 82 & 76 & 68 & -28 & $<0.001$ \\
\hline Breakfast cereal & 35 & 35 & 33 & 32 & 29 & -17 & 0.008 \\
\hline Fish & 17 & 16 & 17 & 16 & 14 & -16 & 0.185 \\
\hline Meat and meat products & 119 & 114 & 113 & 113 & 96 & -20 & $<0.001$ \\
\hline Pasta, rice pizza, other cereal & 66 & 68 & 63 & 58 & 54 & -19 & 0.002 \\
\hline Vegetables including baked beans & 89 & 76 & 72 & 67 & 62 & -31 & $<0.001$ \\
\hline Potatoes and products & 117 & 126 & 127 & 120 & 108 & -8 & 0.029 \\
\hline Fruit & 75 & 74 & 54 & 63 & 56 & -26 & $<0.001$ \\
\hline Fruit juice & 75 & 69 & 63 & 46 & 46 & -38 & $<0.001$ \\
\hline Sugar, preserves and confectionery & 18 & 33 & 40 & 50 & 68 & 273 & $<0.001$ \\
\hline Soft drinks (including squash) & 439 & 567 & 629 & 753 & 885 & 101 & $<0.001$ \\
\hline Biscuits, cakes and pastries & 39 & 43 & 54 & 51 & 55 & 41 & $<0.001$ \\
\hline Puddings, yogurt, ice-cream & 53 & 64 & 67 & 70 & 69 & 31 & $<0.001$ \\
\hline Savoury snacks & 15 & 18 & 17 & 19 & 19 & 31 & $<0.001$ \\
\hline
\end{tabular}

${ }^{\star}$ For details of procedures and quintiles, see Methods and Table 1.

and cakes (g/d adjusted for total EI) were all weakly inversely correlated with zinc intake while breakfast cereals were positively correlated. However, as associations differed between nutrients it is not possible to quantify the impact of soft drinks at this stage.

Studies and reports in the literature vary in their interpretation of dietary sugars' impact on micronutrient intake or dietary quality. Some emphasise the negative direction of most trends, tending to ignore their strength, implying that an optimal diet contains no added sugars at all. Others consider that inverse associations are inconsistent, and weak or unquantifiable, that nutrient intakes are generally adequate, and dilution by sugars is relatively unimportant in terms of public health. However, the balanced view should avoid both over-exaggeration and complacency. The importance of a $10-20 \%$ reduction in nutrient intake hinges on whether intakes are more than adequate or are borderline, but the data and criteria for assessing requirements are themselves an issue of debate. For many nutrients, homeostatic mechanisms affecting absorption and excretion cannot be factored in to estimated requirements, while estimates for zinc requirements in children are 'probably generous'(21).
The EAR cut-point method is a straightforward and accurate measure of the prevalence of inadequate intakes in a population when nutrient requirements are normally distributed and the true prevalence is between about 8 and $92 \%^{(27,31-34)}$. However, when the distribution of requirements is skewed (as for iron in women) the EAR cut-point method tends to underestimate the prevalence of inadequacy ${ }^{(31)}$.

Biochemical measures of status for key nutrients provide a useful means of validating the dietary findings. Reduced levels of transferrin saturation $(<15 \%)$ and serum ferritin $(<15$ or $<20 \mu \mathrm{g} / \mathrm{l})$ are often used to define iron deficiency ${ }^{(35)}$. Serum ferritin is a reliable and sensitive parameter for the assessment of iron stores in healthy subjects and is widely used in clinical practice and screening. Transferrin saturation, the ratio of serum iron to iron-binding capacity, is the most accurate indicator of iron supply to the bone marrow although it has wide diurnal variation and low specificity. In the present study, serum ferritin and transferrin saturation showed a weak inverse association with \%EAS but were more strongly (positively) associated with consumption of meat. Plasma magnesium is regulated by the kidneys but low levels may be due to long-term dietary deficiency, malabsorption or

Table 6. Micronutrient status for selected nutrients according to level of added sugars*

\begin{tabular}{|c|c|c|c|c|c|c|c|c|c|}
\hline & \multirow[b]{2}{*}{$n$} & \multicolumn{5}{|c|}{ Quintiles of added sugars } & \multirow[b]{2}{*}{ Mean } & \multicolumn{2}{|c|}{ Significance tests $†$} \\
\hline & & Q1 & Q2 & Q3 & Q4 & Q5 & & Linear $P$ value & Q1 v. Q5 $P$ value \\
\hline Serum ferritin $(\mu \mathrm{g} / \mathrm{l})$ & 842 & 403 & 343 & 352 & 344 & 336 & 355 & 0.055 & 0.026 \\
\hline Iron saturation (\%) & 925 & $24 \cdot 0$ & $22 \cdot 3$ & $21 \cdot 7$ & $22 \cdot 7$ & $22 \cdot 1$ & 225 & 0.112 & 0.046 \\
\hline $\mathrm{Hb}(\mathrm{g} / \mathrm{l})$ & 1075 & 133 & 132 & 134 & 132 & 133 & 133 & 0.993 & 0.879 \\
\hline Plasma magnesium (mmol/l) & 1030 & 0.92 & 0.93 & 0.92 & 0.92 & 0.92 & 0.92 & 0.363 & 0.862 \\
\hline Plasma zinc $(\mu \mathrm{mol} / \mathrm{l})$ & 786 & $14 \cdot 7$ & $14 \cdot 7$ & $14 \cdot 6$ & $14 \cdot 8$ & 14.5 & $14 \cdot 7$ & 0.463 & 0.288 \\
\hline Plasma $\beta$-carotene $(\mu \mathrm{mol} / \mathrm{l})$ & 983 & $0 \cdot 30$ & 0.33 & 0.32 & 0.31 & 0.31 & 0.32 & 0.623 & 0.593 \\
\hline Plasma $\alpha$-cryptoxanthin $(\mu \mathrm{mol} / \mathrm{l})$ & 983 & 0.053 & 0.061 & 0.057 & 0.064 & 0.063 & 0.060 & 0.003 & 0.002 \\
\hline Plasma $\beta$-cryptoxanthin $(\mu \mathrm{mol} / \mathrm{l})$ & 983 & 0.17 & $0 \cdot 19$ & $0 \cdot 16$ & 0.17 & 0.16 & 0.17 & 0.631 & 0.832 \\
\hline Plasma selenium $(\mu \mathrm{mol} / \mathrm{l})$ & 1029 & 0.90 & 0.88 & 0.87 & 0.86 & 0.83 & 0.87 & $<0.001$ & $<0.001$ \\
\hline
\end{tabular}

*For details of procedures and quintiles, see Methods and Table 1.

† Based on log-transformed values for serum ferritin and carotenoids. 
renal loss ${ }^{(21)}$. Mean plasma magnesium levels in all quintiles were within the normal reference range $(0.75-0.95 \mathrm{mmol} / \mathrm{l})$. Plasma zinc is not a particularly reliable indicator of status but deficiency has been defined as a plasma concentration below $10.7 \mu \mathrm{mol}$ in fasted adults ${ }^{(13,36)}$. Mean zinc levels were well above this level and there was no evidence of a decline with increasing sugars intake. Plasma carotenoids reflect intake in the short and medium term ${ }^{(37)}$. The weak positive association found between $\alpha$-cryptoxanthin and \%EAS is difficult to explain and probably spurious. Finally, plasma selenium was included in the present analysis due to concerns about suboptimal intakes in the population. Although frank selenium deficiency (plasma levels below about $0.11 \mu \mathrm{mol} / \mathrm{l}$ ) is rare, the normal reference range for children aged 4-16 years is $0.7-1.7 \mu \mathrm{mol} / \mathrm{l}^{(25)}$. Plasma selenium was inversely correlated with \%EAS $(r-0.17)$, but positively correlated with consumption of fish, cereal dishes, meat and eggs. Similar associations between selenium status and consumption of fish have recently been reported among British adults ${ }^{(38)}$. Since there is no known biological mechanism directly implicating sugar in low selenium status, high \%EAS is most likely acting as a surrogate marker for a diet lower in protein, particularly fish and meat. Unfortunately, selenium intake data were not included in the NDNS4-18 due to inadequacies in the composition tables.

Interestingly, levels of glutathione peroxidase, the more sensitive indicator of functional selenium status and the criterion for sufficiency adopted in setting most dietary reference values ${ }^{(39)}$, was uncorrelated with \%EAS (data not shown). On the other hand, since current plasma selenium concentrations in the UK do not allow maximal expression of glutathione peroxidase $^{(40)}$, it would be wise to monitor selenium status in general, preferably using more sensitive indices such as glutathione peroxidase and selenoprotein $\mathrm{P}^{(41)}$.

In conclusion, the impact of added sugars on micronutrient intakes appears modest but may have relevance for children who have inadequate micronutrient intakes coupled with a diet high in added sugars (i.e. around $23 \%$ EAS). However, the major determinant of micronutrient adequacy is the consumption of foodstuffs rich in nutrients. Thus an important question posed by this research is how the association between dietary sugars and micronutrient intake is modulated by food choices, both in regard to high-sugar foods and to other foods. Some foods high in added sugar are good sources of micronutrients and have a positive impact on diet quality (e.g. breakfast cereals, yogurts) ${ }^{(12,42)}$. Further work may be warranted to distinguish between sources of added sugars in regard to impact on micronutrient intakes, while statistical modelling could also be used to explore the impact of various dietary change scenarios. A related question is whether advice to reduce added sugars would necessarily improve nutritional status and promote healthy body weight. This cannot be assumed, as diets low in sugar tend to be higher in fat energy and also salt ${ }^{(10)}$, while the jury is still out on whether body weight is influenced by the proportion of dietary carbohydrate or its type ${ }^{(43,44)}$. Interventions are therefore required to test how individuals, and particularly those who are nutritionally disadvantaged, respond in practice when attempting to reduce added sugars intake. Secondly, given the apparent discrepancies between dietary and biochemical assessments of adequacy, these may need to be reviewed.

\section{Acknowledgements}

S. G. is a registered public health nutritionist working as an independent consultant. A. B. is Head of the Sugar Bureau and a registered dietitian and registered nutritionist. S. G. was responsible for study design, data analysis, and writing and revising the paper. A. B. assisted with protocol design and critically reviewed the paper. This secondary analysis of NDNS data was funded by a grant from the Sugar Bureau (UK). The NDNS4-18 was commissioned and funded jointly by the UK Ministry of Agriculture, Fisheries and Food and the Department of Health and obtained under licence from the UK Data Archive. We are grateful to Professor Margaret Rayman, University of Surrey, UK, for helpful discussions on selenium nutrition.

\section{References}

1. Gibney M, Sigman-Grant M, Stanton JL Jr \& Keast DR (1995) Consumption of sugars. Am J Clin Nutr 62, 78S-193S.

2. Bolton-Smith C (1996) Intake of sugars in relation to fatness and micronutrient adequacy. Int J Obes Relat Metab Disord 20, Suppl. 2, S31-S33.

3. Ruxton CH, Garceau FJ \& Cottrell RC (1999) Guidelines for sugar consumption in Europe: is a quantitative approach justified? Eur J Clin Nutr 53, 503-513.

4. Ruxton $\mathrm{CH}$ (2003) Dietary guidelines for sugar: the need for evidence. Br J Nutr 90, 245-247.

5. Murphy SP \& Johnson RK (2003) The scientific basis of recent US guidance on sugars intake. Am J Clin Nutr 78, 827S-833S.

6. Rennie KL \& Livingstone MB (2007) Associations between dietary added sugar intake and micronutrient intake: a systematic review. Br J Nutr 97, 832-841.

7. Gibson S (2007) Dietary sugars intake and micronutrient adequacy - a review of the evidence. Nutr Res Rev 20, 121-131.

8. Gibson S (1993) Consumption and sources of sugars in the diets of British schoolchildren: are high-sugar diets nutritionally inferior? J Hum Nutr Diet 6, 355-371.

9. Gibson SA (1997) Do diets high in sugars compromise micronutrient intakes? Micronutrient intakes in the Dietary and Nutritional Survey of British Adults according to dietary concentration of 'added', 'non-milk extrinsic' or 'total' sugars. J Hum Nutr Diet 10, 125-133.

10. Gibson SA (1997) Non-milk extrinsic sugars in the diets of preschool children: association with intakes of micronutrients, energy, fat and NSP. Br J Nutr 78, 367-378.

11. Gibson S (2001) Dietary sugars and micronutrient dilution in normal adults aged 65 years and over. Public Health Nutr 4, 1235-1244.

12. Gibson S (2003) Micronutrient intakes, micronutrient status and lipid profiles among young people consuming different amounts of breakfast cereals: further analysis of data from the National Diet and Nutrition Survey of Young People aged 4 to 18 years. Public Health Nutr 6, 815-820.

13. Gregory J \& Lowe S (2000), National Diet and Nutrition Survey: Young People Aged 4 to 18 Years, vol. 1: Report of the Diet and Nutrition Survey. London: HMSO.

14. Child Growth Foundation. LMS software for calculating BMI $z$-score from UK reference curves. http://www.childgrowthfoundation.org

15. Bellizzi MC \& Dietz WH (1999) Workshop on childhood obesity: summary of the discussion. Am J Clin Nutr 70, 173S-175S.

16. Cole TJ, Bellizzi MC, Flegal KM \& Dietz WH (2000) Establishing a standard definition for child overweight and obesity worldwide: international survey. Br Med J 320, 1240-1245. 
17. Dietz WH \& Robinson TN (1998) Use of the body mass index (BMI) as a measure of overweight in children and adolescents. J Pediatr 132, 191-193.

18. Gibson S \& Neate D (2007) Sugar intake, soft drink consumption and body weight among British children: further analysis of National Diet and Nutrition Survey data with adjustment for under-reporting and physical activity. Int J Food Sci Nutr 58, $445-460$.

19. Goldberg GR, Black AE, Jebb SA, Cole TJ, Murgatroyd PR, Coward WA \& Prentice AM (1991) Critical evaluation of energy intake data using fundamental principles of energy physiology: 1. Derivation of cut-off limits to identify underrecording. Eur J Clin Nutr 45, 569-581.

20. Black AE (2000) The sensitivity and specificity of the Goldberg cut-off for EI:BMR for identifying diet reports of poor validity. Eur J Clin Nutr 54, 395-404.

21. Department of Health (1991) Dietary Reference Values for Food Energy and Nutrients for the United Kingdom. Report on Health and Social Subjects no. 41. London: HMSO.

22. Murphy SPB \& Susan I (2005) Challenges in using the dietary reference intakes to plan diets for groups. Nutr Rev 63, 267-271.

23. Institute of Medicine (2002) Dietary Reference Intakes for Energy, Carbohydrate, Fat, Fibre, Fatty Acids, Cholesterol, Protein and Amino Acids. Washington, DC: Food and Nutrition Board.

24. Department of Health (1989) Dietary Sugars and Human Disease. London: HMSO.

25. Scottish Trace Element and Micronutrient Reference Laboratory (2007) Reference values. http://www.trace-elements.org.uk/ Selenium.htm

26. Bolton-Smith C \& Woodward M (1995) Antioxidant vitamin adequacy in relation to consumption of sugars. Eur $J$ Clin Nutr 49, 124-133.

27. Rutishauser IH \& Mackerras DE (2003) Reliable estimates of dietary adequacy in population sub-groups depend on appropriate analysis of national dietary survey data. Asia Pac J Clin Nutr 12, S23.

28. Forshee RA \& Storey ML (2004) Controversy and statistical issues in the use of nutrient densities in assessing diet quality. J Nutr 134, 2733-2737.

29. Alexy U, Sichert-Hellert W \& Kersting M (2003) Associations between intake of added sugars and intakes of nutrients and food groups in the diets of German children and adolescents. Br J Nutr 90, 441-447.

30. Overby NC, Lillegaard IT, Johansson L \& Andersen LF (2004) High intake of added sugar among Norwegian children and adolescents. Public Health Nutr 7, 285-293.
31. Institute of Medicine Food and Nutrition Board (2000) Dietary Reference Intakes: Applications in Dietary Assessment. Washington, DC: National Academies Press.

32. Institute of Medicine Food and Nutrition Board (2000) Dietary Reference Intakes: Applications in Dietary Planning. Washington, DC: National Academies Press.

33. Hannon EM, Kiely M, Harrington KE, Robson PJ, Strain JJ \& Flynn A (2001) The North/South Ireland Food Consumption Survey: mineral intakes in 18-64-year-old adults. Public Health Nutr 4, 1081-1088.

34. Beaton GH (1994) Criteria of an adequate diet. In Modern Nutrition in Health and Disease, 8th ed., pp. 1491-1505 [MEOJ Shils and M Shike, editors]. Philadelphia, PA: Lea \& Febiger.

35. Institute of Medicine (1993) Iron Deficiency Anemia: Recommended Guidelines for the Prevention, Detection, and Management Among U.S. Children and Women of Childbearing Age. Washington, DC: National Academy of Sciences.

36. Pilch SM \& Senti FR (1984) Assessment of Zinc Nutritional Status of the US Population Based on Data Collected in the Second National Health and Nutrition Examination Survey (NHANES II) 1976-1980. Bethesda, MD: LSRO. Federation of American Societies for Experimental Biology.

37. Basu T \& Dickerson J (1996) Vitamins in Human Health and Disease. Wallingford, UK: CAB International.

38. Bates CJ, Prentice A, Birch MC \& Delves HT (2007) Dependence of blood indices of selenium and mercury on estimated fish intake in a national survey of British adults. Public Health Nutr 10, 508-517.

39. Thomson CD (2004) Assessment of requirements for selenium and adequacy of selenium status: a review. Eur J Clin Nutr 58, 391-402.

40. Rayman MP (2002) The argument for increasing selenium intake. Proc Nutr Soc 61, 203-215.

41. Burk RF \& Hill KE (2005) Selenoprotein P: an extracellular protein with unique physical characteristics and a role in selenium homeostasis. Annu Rev Nutr 25, 215-235.

42. Frary CD, Johnson RK \& Wang MQ (2004) Children and adolescents' choices of foods and beverages high in added sugars are associated with intakes of key nutrients and food groups. J Adolesc Health 34, 56-63.

43. Vermunt SHF, Pasman WJ, Schaafsma G \& Kardinaal AFM (2007) Effects of sugar intake on body weight: a review. Obes Rev 4, 91-99.

44. van Dam RM \& Seidell JC (2007) Carbohydrate intake and obesity. Eur J Clin Nutr 61, S75-S99. 